Kragujevac Journal of Mathematics

Volume 42(4) (2018), Pages 495-503.

\title{
FAULT-TOLERANT METRIC DIMENSION PROBLEM: A NEW INTEGER LINEAR PROGRAMMING FORMULATION AND EXACT FORMULA FOR GRID GRAPHS
}

\author{
ANA SIMIĆ ${ }^{1}$, MILENA BOGDANOVIĆ2 ${ }^{2}$ ZORAN MAKSIMOVIĆ ${ }^{3}$, \\ AND JELISAVKA MILOŠEVIĆ ${ }^{4}$
}

\begin{abstract}
In this paper, fault-tolerant metric dimension problem (FTMDP) is considered. The existing integer linear programing (ILP) formulation, from the literature is improved, using lesser number of variables and constraints. Correctness proof shows that improved linear programing formulation is equivalent to the existing one. Computational results on random graphs proposed for similar problems in the literature, clearly show the advantage of a new ILP formulation. Additionally, the exact value of fault-tolerant metric dimension of grid graphs are given and proved.
\end{abstract}

\section{INTRODUCTION}

The metric dimension problem was introduced independently by Slater (1975) [10] and Harary and Melter (1976) [4]. Shortly, the metric dimension of an undirected and connected graph $G$ is the minimum cardinality of a subset $S$ of vertex set $V$ of $G$ with the property that all the vertices in $V$ are uniquely determined by their distances to the vertices in $S$.

One of most interesting application of the metric dimension problem arises in robot navigation [8]. Let a robot is navigating in a space modeled by a graph $G$ and it wants to know its current position. Robot usually send a signal to find out how far it is form each among a set of fixed vertices which we call landmarks. The problem of determining the minimum number of landmarks and their positions such that the robot can always uniquely determine its location is equivalent to the metric dimension problem.

Key words and phrases. Integer linear programming, fault-tolerant metric dimension. 2010 Mathematics Subject Classification 2010. Primary: 90C10. Secondary: 05C12, 68R10. Received: January 11, 2016.

Accepted: May 08, 2017. 
If one of landmarks does not work properly, we will not have enough information for a robot to uniquely determine its location. In order to overcome this kind of problems, concept of fault-tolerant metric dimension was introduced by Hernando et al. (2008) [5]. Fault-tolerant resolving set provide correct information even when one of the landmarks is not working. Shortly, a resolving set is said to be fault-tolerant if the removal of any element from it keeps it resolving. The fault-tolerant metric dimension (FTMD) of $G$ is the minimum cardinality of a fault-tolerant resolving set, denoted by $\beta^{\prime}(G)$.

\section{Previous Work}

Javaid et al. (2009) [7] were presented that for every pair $a, b \in \mathbb{N}, a \neq b-1$ and $5 \leq a \leq b$, is realizable as the fault-tolerant metric dimension and the fault-tolerant partition dimension of some connected graphs. Also, bounds of maximum order of a graph $G$ was presented in terms of its diameter, fault-tolerant metric dimension and the fault-tolerant partition dimension.

In [2] was shown that for every pair $a, b \in \mathbb{N}$ with $b \geq 6$ and $\left\lfloor\frac{b}{2}\right\rfloor+1 \leq a \leq b-2$ is realizable as the fault-tolerant metric dimension and the fault-tolerant partition dimension of some connected graphs. Also, it was given the classification when a fault-tolerant partition dimension of graph is equal to its order, i.e., $\beta^{\prime}(G)=|G|$.

In [6] were shown that every pair $a, b \in \mathbb{N}$ with $a \leq b, 2 \cdot a \neq b+3$, and $2 \cdot a \geq b+2$ is realizable as the fault-tolerant metric dimension and the fault-tolerant partition dimension of some connected graphs. Moreover, in [6] some theoretical properties of weak total metric dimension and strong total metric dimension of $\mathrm{G}$ were presented.

Azhar (2015) [1] was found the fault-tolerant metric dimension of 4-regular family of Harary graphs $H(4, n)$, for all $n \geq 8$. He was proved that this was a family of graphs with constant fault-tolerant metric dimension, i.e., independent of the choice of graphs in the family. Moreover, the metric dimension, the total metric dimension and the weak total metric dimension of those graphs were found.

\section{Existing Mathematical Formulations}

3.1. Basic mathematical formulation. Let a simple connected undirected graph $G=(V, E)$, where $V=\{1,2, \ldots, n\}$ and $|E|=m$. It is easy to determine the length $d(u, v)$ of a shortest $u-v$ path for all $u, v \in V$ using any shortest path algorithm. A vertex $p$ of the graph $G$ is said to resolve (distinguish) two vertices $u$ and $v$ of $G$ if $d(u, p) \neq d(v, p)$. An ordered vertex set $S=\left\{s_{1}, s_{2}, \ldots, s_{k}\right\}$ of $G$ is a fault-tolerant resolving set of $G$, if for each vertex $p \in S$, every two distinct vertices $u, v \in V$ of $G$ are resolved by some vertex of $S \backslash\{p\}$. A fault-tolerant metric basis of $G$ is a fault-tolerant resolving set of the minimum cardinality. The fault-tolerant metric dimension of $G$, denoted by $\beta^{\prime}(G)$, is the cardinality of its fault-tolerant metric basis. 
3.2. Existing ILP formulation. In this subsection it will be presented existing integer linear programing formulation from [9]. The coefficient matrix A is defined as follows:

$$
A_{(u, v),(i, j)}= \begin{cases}1, & d(u, i) \neq d(v, i) \text { and } d(u, j) \neq d(v, j) \\ 0, & d(u, i)=d(v, i) \text { or } d(u, j)=d(v, j),\end{cases}
$$

where $1 \leq u<v \leq n, 1 \leq i<j \leq n$. Variable $x_{i}$ described by (3.2) determines whether vertex $i$ belongs to a set $S$. Similarly, variable $y_{i j}$ described by (3.3) determines whether both $i, j$ are in $S$, that is

$$
\begin{gathered}
x_{i}= \begin{cases}1, & i \in S, \\
0, & i \notin S,\end{cases} \\
y_{i j}= \begin{cases}1, & i, j \in S, \\
0, & \text { otherwise. }\end{cases}
\end{gathered}
$$

The ILP model of the FTMD problem from [9] can now be formulated as:

$$
\min \sum_{i=1}^{n} x_{i}
$$

subject to:

$$
\begin{aligned}
\sum_{i=1}^{n-1} \sum_{j=i+1}^{n} A_{(u, v),(i, j)} \cdot y_{i j} \geq 1, & 1 \leq u<v \leq n, \\
y_{i j} \leq \frac{1}{2} x_{i}+\frac{1}{2} x_{j}, & 1 \leq i<j \leq n, \\
y_{i j} \geq x_{i}+x_{j}-1, & 1 \leq i<j \leq n, \\
y_{i j} \in\{0,1\}, x_{k} \in\{0,1\}, & 1 \leq i<j \leq n, 1 \leq k \leq n .
\end{aligned}
$$

The objective function (3.4) represents the minimal cardinality of a set $S$. Constraints (3.5)-(3.7) make sure that for each two vertices $u$ and $v$ there exists at least two vertices from set $S$ which resolves $u$ and $v$, i.e., $S$ is a fault-tolerant resolving set. Constraints (3.8) reflect the binary nature of decision variables $x_{i}$ and $y_{i j}$.

\section{A New Integer Linear Programing Formulation}

In order to give a new improved ILP model we must change matrix $A_{(u, v),(i, j)}$ defined by (3.1) into the new matrix $A_{(u, v), i}^{\prime}$ :

$$
A_{(u, v), i}^{\prime}= \begin{cases}1, & d(u, i) \neq d(v, i) \\ 0, & d(u, i)=d(v, i)\end{cases}
$$


Now, constraints (3.5)-(3.8) is replaced by new ones

$$
\begin{gathered}
\sum_{i=1}^{n} A_{(u, v), i}^{\prime} \cdot x_{i} \geq 2, \quad 1 \leq u<v \leq n, \\
x_{k} \in\{0,1\}, \quad 1 \leq k \leq n .
\end{gathered}
$$

Therefore, a new ILP formulation of FTMDP contains objective function (3.4) subject to (4.2) and (4.3). The equivalence of a new ILP formulation with original mathematical formulation of the FTMDP is given in following proposition.

Proposition 1. Set $S$ is a fault-tolerant resolving set of $G$ of minimal cardinality if and only if constraints (3.4), (4.2) and (4.3) are satisfied.

Proof. $(\Rightarrow)$ Suppose that $S$ is a fault-tolerant resolving set, then $S$ is also a resolving set. Let we define $x_{i}$ as in (3.2). Then, constraints (4.3) are satisfied by default. Let we fix $u$ and $v$ such that $1 \leq u<v \leq n$. Since $S$ is a resolving set then there exist $p \in S$ such that $d(u, p) \neq d(v, p)$. Additionally, $S$ is a fault-tolerant resolving set, so for vertex $p \in S$ it holds $(\exists q \in S \backslash\{p\}) d(u, q) \neq d(v, q)$. Therefore, it holds $A_{(u, v), p}^{\prime}=A_{(u, v), q}^{\prime}=1$ and $x_{p}=x_{q}=1$, so $\sum_{i=1}^{n} A_{(u, v), i}^{\prime} \cdot x_{i} \geq 2$, which means that constraints (4.2) are satisfied. Since $\sum_{i=1}^{n} x_{i}=|S|$, it holds that minimal objective function of ILP model (3.4), (4.2), (4.3) is less or equal than $|S|$.

$(\Leftarrow)$ Let we define set $S=\left\{i \mid x_{i}=1\right\}$. By constraints (4.2) and binary nature of variables $x_{i}$ which are ensured by constraints (4.3), it holds that for each $1 \leq u<v \leq n$ there exist at least two vertices $i_{1}$ and $i_{2}$ for which it is $A_{(u, v), i_{1}}^{\prime}=A_{(u, v), i_{2}}^{\prime}=1$ and $x_{i_{1}}=x_{i_{2}}=1$, or equivalently $d\left(u, i_{1}\right) \neq d\left(v, i_{1}\right), d\left(u, i_{2}\right) \neq d\left(v, i_{2}\right), i_{1} \in S$ and $i_{2} \in S$. Therefore, it is obvious that if we exclude arbitrary member from set $S$, at least one member of remainder of $S$ resolve pair $u$ and $v$. Therefore, $S$ is a fault-tolerant resolving set, with cardinality equal to $\sum_{i=1}^{n} x_{i}$, so it follows that fault-tolerant resolving set with minimal cardinality has at most $\sum_{i=1}^{n} x_{i}$ elements.

Note that, the new ILP model (3.4), (4.2) and (4.3) has only $n$ binary variables and $\left(\begin{array}{l}n \\ 2\end{array}\right)$ constraints which is much smaller compared to ILP model (3.4)-(3.8) from [9], which has $\left(\begin{array}{l}n \\ 2\end{array}\right)+n$ binary variables and $3 \cdot\left(\begin{array}{l}n \\ 2\end{array}\right)$ constraints.

\section{GRID GraphS}

For this class of graphs $G R(a, b)$, vertices can be represented as $V=\{(i, j) \mid$ $1 \leq i \leq a, 1 \leq j \leq b\}$ and each vertex $v=(i, j)$ has at least two and at most four neighbours: $(i-1, j)$ for $i>1,(i+1, j)$ for $i<a,(i, j-1)$ for $j>1$ and $(i, j+1)$ for $j<b$.

In the sequel there will be used one trivial property of grid graphs. 
Property 1. For grid graph and every two vertices $u, v \in V$, and vertex $w$ such that $d(v, w)=1$ it holds $d(u, w)=d(u, v)+1$ or $d(u, w)=d(u, v)-1$.

Now, we will give the exact value of fault-tolerant metric dimension on grid graphs.

Theorem 5.1. For $a, b \in \mathbb{N}, a \leq b$, holds

$$
\beta^{\prime}(G R(a, b))= \begin{cases}\text { undefined, } & a=b=1, \\ 2, & a=1, b \geq 2, \\ 4, & a \geq 2 .\end{cases}
$$

Proof. $\underline{a=b=1}$. Graph $G R(1,1)$ is trivial graph with one vertex without edges, so it obviously have no fault-tolerant resolving set which must have at least two elements. Therefore $\beta^{\prime}(G R(1,1))$ is undefined.

$a=1, b \geq 2$. Obviously $G R(1, b) \cong P_{b}$, where $P_{b}$ is a path with $b$ vertices. Easily, set $S=\{1, b\}$ is fault-tolerant resolving set, since

$$
i \neq j \Rightarrow(d(i, 1)=i-1 \neq j-1=d(j, 1) \wedge d(i, b)=b-i \neq b-j=d(j, b)) .
$$

Since fault-tolerant metric dimension is at least 2 , it holds $\beta^{\prime}(G R(1, b))=\beta^{\prime}\left(P_{b}\right)=2$. $a \leq b, a \geq 2$, Upper bound. We prove that set $S=\{(1,1),(1, b),(a, 1),(a, b)\}$ is a fault-tolerant resolving set. Let $u=\left(i_{u}, j_{u}\right)$ and $v=\left(i_{v}, j_{v}\right)$ are two different arbitrary vertices from $G R(a, b)$.

- Case 1: $i_{u}+j_{u} \neq i_{v}+j_{v}$. In this case, $d(u,(1,1))=i_{u}+j_{u}-2 \neq i_{v}+j_{v}-2=$ $d(v,(1,1))$ and $d(u,(a, b))=a+b-i_{u}-j_{u} \neq a+b-i_{v}-j_{v}=d(v,(a, b))$.

- Case 2: $i_{u}-j_{u} \neq i_{v}-j_{v}$. In this case, $d(u,(1, b))=b-1+i_{u}-j_{u} \neq b-1+i_{v}-j_{v}=$ $d(v,(1, b))$ and $d(u,(a, 1))=a-1-i_{u}+j_{u} \neq a-1-i_{v}+j_{v}=d(v,(a, b))$.

- Case 3: $i_{u}+j_{u}=i_{v}+j_{v}$ and $i_{u}-j_{u}=i_{v}-j_{v}$. Easily, by summation and subtraction, we have $i_{u}=i_{v}$ and $j_{u}=j_{v}$, which is in contradiction with premise that $u$ and $v$ are different.

From Case 1 and Case 2, it is evident that set $S$ is a fault-tolerant resolving set of $G R(a, b)$.

$a \leq b, a \geq 2$, Lower bound. Now, we must prove that fault-tolerant resolving set of grid graph in this case have cardinality at least 4 . Suppose the contrary, that exists fault-tolerant resolving set of cardinality equal to 2 or 3 , and prove the contradiction. Let in case of $|S|=2$ denote $S=\{p, q\}$, while for $|S|=3$ denote $S=\{p, q, r\}$ with $p=\left(i_{p}, j_{p}\right), q=\left(i_{q}, j_{q}\right)$ and $r=\left(i_{r}, j_{r}\right)$.

We have the following cases.

- Case 1: $|S|=2$. Since all vertices in grid graph have at least two neighbours, let $u, v \in V, u \neq v$, be the neighbours of $p$. Therefore, $d(u, p)=d(v, p)$ so $S=\{p, q\}$ is not fault-tolerant resolving set, since if we omit vertex $q$, it not distinguish all vertices from $V$.

- Case 2: $|S|=3$ and at least one $i_{p}, i_{q}, i_{r}$ is diferent from 1 and diferent from $a$, or at least one $j_{p}, j_{q}, j_{r}$ is diferent from 1 and diferent from $b$. Without loss of generality we can presume $i_{p} \neq 1 \wedge i_{p} \neq a$. In that case vertex $p$ has 3 or 
4 neighbours. By Property 1, there are two neighbours of $p$ named $u$ and $v$, such that $d(u, q)=d(v, q)=d(p, q)+1$ or $d(u, q)=d(v, q)=d(p, q)-1$. Since, there exist two vertices $u$ and $v$ such that $d(u, p)=d(v, p)$ and $d(u, q)=d(v, q)$, then $S$ is obviously not a fault-tolerant resolving set.

- Case 3: $|S|=3$ and all $i_{p}, i_{q}, i_{r}$ is equal to 1 or equal to $a$, and all $j_{p}, j_{q}, j_{r}$ is equal to 1 or equal to $b$. Without loss of generality we can presume $r=(1,1)$, $q=(1, b)$ and $p=(a, 1)$. Let $u=(a-1,1)$ and $v=(a, 2)$. Then $d(u, p)=$ $d(v, p)=1$ and $d(u, q)=d(v, q)=d(p, q)-1$. Once again, there exist two vertices $u$ and $v$ such that $d(u, p)=d(v, p)$ and $d(u, q)=d(v, q)$, then $S$ is obviously not a fault-tolerant resolving set.

In all three cases we have contradiction with starting premise $|S| \leq 3$. Since it is already proved that for $a \geq 1$ it holds $\beta^{\prime}(G R(a, b)) \leq 4$, then for $a \geq 1$ fault-tolerant metric dimension of $G R(a, b)$ is equal to 4.

\section{Computational Results}

In this section computational results and direct comparison between new and existing ILP formulation will be presented. All computations were executed on AMD FX-8300, 3.3 GHz PC with 4GB RAM using single core. The both ILP models were coded in CPLEX 12.6 solver using the $\mathrm{C}$ programming language.

Random instances from [3], with 50 and 100 vertices, were used for testing. This set of instances contains overall 36 graphs, from sparse to dense ones. The time limit for CPLEX solver on each model is set to two hours (7200 seconds). If CPLEX does not finish work and prove optimality in that time interval, running is stopped and partial results of solution value and lower bound is reported.

Table 1 contains experimental data for instances whose optimal solution is proven by CPLEX solver with at least one model. In the first column the name of an instance is given, while second and third columns represent the number of vertices and the number of edges. The fourth column is labeled with $O p t$ and contains corresponding optimal solution value. Next three columns represent the data obtained by CPLEX on existing model from [9]:

- the fifth column is labeled as Sol and contains the obtained result, with notation opt if the optimal solution is proved or value with asteriks if it is only reached;

- the sixth column labeled by $L B$ contains the lower bound if the optimal solution is not proved, while otherwise it is blank;

- next column, labeled by $t$ presents total running time in seconds.

Last three columns represent the data obtained by CPLEX on the new model, presenting in the same way as for existing ILP model.

Note that we present original lower bounds given by CPLEX solver. Since the objective function value is integer, each non-integer lower bound can be replaced by first integer greater or equal than it. 
TABLE 1. Results on instances with known optimal solution

\begin{tabular}{|c|c|c|c|c|c|c|c|c|}
\hline \multirow[t]{2}{*}{ Inst. } & \multirow[t]{2}{*}{$|\mathrm{V}|$} & \multirow[t]{2}{*}{$\bar{E} \mid$} & \multirow[t]{2}{*}{$O p t$} & \multicolumn{3}{|c|}{ ILP from $[9]$} & \multicolumn{2}{|c|}{ New ILP } \\
\hline & & & & Sol & $L B$ & $t[\mathrm{sec}]$ & Sol & $t[s e c]$ \\
\hline Random-50-1 & 50 & 49 & 11 & opt & & 0.625 & opt & 0.015 \\
\hline Random-50-2 & 50 & 49 & 6 & opt & & 0.937 & ор⿰ & 0.031 \\
\hline Random-50-3 & 50 & 58 & 18 & opt & & 0.796 & opt & 0.015 \\
\hline Random- & 50 & 54 & 12 & opt & & 0.640 & opt & 0.01 \\
\hline Random-50-5 & 50 & 67 & 9 & opt & & 1.609 & opt & 0.031 \\
\hline Random-50-6 & 50 & 86 & 7 & opt & & 1007 & opt & $0 \cap 0$ \\
\hline Rand & 50 & 84 & 6 & $6^{*}$ & 2.3019 & 7200 & opt & 0.921 \\
\hline Random-50-8 & 50 & 95 & 6 & $6^{*}$ & 2.3805 & 5623 & opt & 1.171 \\
\hline Random- & 50 & 108 & 6 & 7 & 2.2462 & 7200 & opt & 2.218 \\
\hline Random- & 50 & 112 & 7 & $7^{*}$ & 2.2978 & 6230 & opt & 2.2 \\
\hline $0-20$ & 50 & 248 & 10 & 11 & 2.4616 & 7200 & opt & 104.2 \\
\hline-30 & 50 & 373 & 10 & 11 & 2.7286 & 7200 & opt & 62.7 \\
\hline $0-40$ & 50 & 475 & 10 & 11 & 2.1928 & 7200 & opt & 291.1 \\
\hline Rand & 50 & 597 & 9 & 10 & 2 & 7200 & opt & 90.2 \\
\hline Rand & 50 & 739 & 10 & $10^{*}$ & 2 & 7200 & opt & 300 \\
\hline$n-50-70$ & 50 & 860 & 10 & 12 & 2.1917 & 7200 & opt & 23.5 \\
\hline $0-80$ & 50 & 980 & 13 & $13^{*}$ & 4.247 & 7200 & opt & 29.7 \\
\hline Random-: & 50 & 1103 & 18 & 20 & 11.1306 & 5103 & opt & 0.65 \\
\hline$\overline{\text { Random- }}$ & 100 & 100 & 11 & opt & & 9.656 & opt & 0.156 \\
\hline $00-2$ & 100 & 109 & 18 & opt & & 9.218 & opt & 0.171 \\
\hline Random-100-3 & 100 & 181 & 8 & 9 & & 7200 & opt & 2.29 \\
\hline Random-100-4 & 100 & 206 & 7 & 100 & 0.1598 & 3057 & opt & 50.76 \\
\hline Random-100-5 & 100 & 231 & 8 & 100 & 0.2244 & 3316 & opt & 162 \\
\hline
\end{tabular}

As it can be seen from Table 1, CPLEX based on existing ILP model from [9] was able, within two hour time limit, to prove optimality of 6 out of 18 instances with 50 vertices, and 2 out of 18 instances with 100 vertices. It additionally reach optimal solutions in 5 cases for instances with 50 vertices. On the other hand, CPLEX based on new ILP model was able within two hour time limit, to prove optimality of all 18 instances with 50 vertices, and 5 out of 18 instances with 100 vertices.

Running times of CPLEX based on existing ILP model is in all cases significantly larger than running times of CPLEX based on new ILP model. For example, for Random-50-6 instance, CPLEX based on existing ILP model prove optimality in 1007 seconds while CPLEX based on new ILP model for that needs only 0.093 seconds.

Table 2 contain the experimental data for the instances with unknown optimal solution. The meaning of all columns is the same as in Table 1, except that column Opt is omitted, since optimal solution is not known.

As it can be seen from Table 2, CPLEX based on new ILP model again produce much better results than CPLEX based on existing ILP model, both in quality of 
TABLE 2. Results on instances with unknown optimal solution

\begin{tabular}{|l|rr|rrr|rrr|}
\hline Inst. & $|\mathrm{V}|$ & $|\mathrm{E}|$ & \multicolumn{3}{|c|}{ ILP from $[9]$} & \multicolumn{3}{|c|}{ New ILP } \\
& & & Sol & LB & $t[$ sec $]$ & Sol & LB & $t[\mathrm{sec}]$ \\
\hline Random-100-6 & 100 & 321 & 11 & 0.1693 & 7200 & 9 & 6.4878 & 3192 \\
Random-100-7 & 100 & 317 & 11 & 0.1843 & 7200 & 9 & 6.6993 & 2765 \\
Random-100-8 & 100 & 398 & 12 & 0.1790 & 7200 & 10 & 6.4962 & 1984 \\
Random-100-9 & 100 & 430 & 11 & 0.1455 & 7200 & 10 & 6.3796 & 1575 \\
\hline Random-100-10 & 100 & 498 & 12 & 0.1697 & 7200 & 11 & 6.8225 & 4862 \\
Random-100-20 & 100 & 981 & 19 & 0.4291 & 7200 & 16 & 9.6572 & 2710 \\
Random-100-30 & 100 & 1477 & 16 & 0.2517 & 7200 & 13 & 8.5100 & 7200 \\
Random-100-40 & 100 & 1945 & 14 & 0.1782 & 7200 & 12 & 7.1708 & 2593 \\
Random-100-50 & 100 & 2483 & 13 & 0.1610 & 7200 & 12 & 7.0782 & 7200 \\
\hline Random-100-60 & 100 & 2985 & 14 & 0.1803 & 7200 & 12 & 7.4088 & 7200 \\
Random-100-70 & 100 & 3435 & 15 & 0.2547 & 7200 & 13 & 8.3824 & 5733 \\
Random-100-80 & 100 & 3935 & 20 & 0.7110 & 7200 & 17 & 10.5280 & 1547 \\
Random-100-90 & 100 & 4446 & 32 & 1.9505 & 7200 & 24 & 18.5498 & 1591 \\
\hline
\end{tabular}

obtained result and quality of obtained lower bound. For example, for Random-100-90 instance, CPLEX based on new ILP model obtain solution of value 24 with lower bound $\lceil 18.5498\rceil=19$, while CPLEX based on existing ILP model obtain solution of value only 32 with much worse lower bound equal to $\lceil 1.9505\rceil=2$.

\section{Conclusions}

This paper is devoted to the fault-tolerant metric dimension problem. Exact results of fault-tolerant metric dimension on grid graphs are given and proved. Proposing the new integer linear programing formulation with much less variables and constraints comparing to the formulation from literature, provide a significant memory savings for solving current problem. Formal proof that a new model is equivalent to the existing one, is also presented. From computational results it is evident, not only theoretical advantage of a new model, but also practical improvements of the computational efforts for solving present problem.

Future work can be directed to designing an exact method using proposed ILP formulation. Other direction of future work can be solving some other similar graph problems.

\section{REFERENCES}

[1] M. N. Azhar, Fault tolerance and total resolvability in regular graphs, Tech. Rep., Researchgate:279529420, 2015, doi:10.13140/RG.2.1.4232.3049.

[2] M. A. Chaudhry, I. Javaid and M. Salman, Fault-tolerant metric and partition dimension of graphs, Util. Math. 83 (2010), 187-199.

[3] V. Currò, The Roman domination problem on grid graphs, Ph.D. thesis, Università di Catania, 2014. 
[4] F. Harary and R. A. Melter, On the metric dimension of a graph, Ars Combin. 2 (1976), 191-195.

[5] C. Hernando, M. Mora, P. Slater and D. Wood, Fault-tolerant metric dimension of graphs, in: International Workshop on Convexity in Discrete Structures (Victor Chepoi 22/03/200602/04/2003), International Press, Somerville, Massachusetts, USA, 2008, pp. 81-85.

[6] I. Javaid, F. Iftikhar and M. Salman, Total resolvability in graphs, Middle-East Journal of Scientific Research 11 (2012), 1649-1658.

[7] I. Javaid, M. Salman, M. A. Chaudhry and S. Shokat, Fault-tolerance in resolvability, Util. Math. 80 (2009), 263-275.

[8] B. R. S. Khuller and A. Rosenfeld, Landmarks in graphs, Discrete Appl. Math. 70 (1996), 217-229.

[9] M. Salman, I. Javaid and M. A. Chaudhry, Minimum fault-tolerant, local and strong metric dimension of graphs, Tech. Rep., arXiv preprint arXiv:1409.2695, 2014.

[10] P. Slater, Leaves of trees, Congr. Numer. 14 (1975), 549-559.

${ }^{1}$ Faculty of Mathematics,

UNIVERSITY OF BELGRADE,

Studentski trg 16, 11000 Belgrade, Serbia

Email address: simicana4as@gmail.com

${ }^{2}$ Pedagogical Faculty,

UNIVERSITY OF Niš,

PARTizanska 14, 17500 VRAnJe, Serbia

Email address: mb2001969@beotel.net

${ }^{3}$ Military ACADEMY,

UNIVERSITY OF DEFENCE,

Generala Pavla Jurišića Šturma 33, 11000 Belgrade, Serbia

Email address: zoran.maksimovic@gmail.com

${ }^{4}$ FASPER,

UNIVERSITY OF BELGRADE,

Visokog Stevana 2, 11000 Belgrade, Serbia

Email address: jelisavkam@gmail.com 